\title{
Ferramenta para o monitoramento dos padrões de teleconexão na América do Sul
}

TOOL FOR MONITORING teleCONNECTION PATtERNS In SOUTH America

\author{
Christie Andre de Souza ${ }^{1}$, Michelle Simões Reboita ${ }^{2}$ \\ 1 - Graduando, Instituto de Recursos Naturais, Universidade Federal de Itajubá, Itaudbá, MG, Brasil \\ 2 - Doutora, Instituto de Recurso Naturais, Universidade Federal de ltajubá, Itauubá, MG, Brasil. \\ EMAIL: CHRISTIE@UNIFEI.EDU.BR, REBOITA@UNIFEI.EDU.BR
}

\begin{abstract}
Teleconnections are a mechanism of natural climate variability. They are associated with local factors that cause climate anomalies in remote sectors of the globe. Since the seasonal climate forecast depends on knowledge of teleconnection patterns, the objective of this study was to present the development of an online tool that gathers a huge amount of information for easy access by users who are interested in monitoring different teleconnection patterns. To achieve this, climate indices made available by different meteorological centers are used. Moreover, two new indices were built and implemented. The methodology relies on the use of web programming language for its implementation and to promote the visualization of climate indices, maps of atmospheric variables, animations, statistical analyses, as well as other products. This tool is available at: https://meteorologia.unifei.edu.br/teleconexoes/. Finally, it should be noted that the tool was designed to be automatically updated each month.
\end{abstract}

Resumo: As teleconexões são um mecanismo de variabilidade natural do clima. Elas estão associadas a fatores locais que causam anomalias climáticas em setores remotos do globo. Como a previsão climática sazonal depende do conhecimento dos padrões de teleconexão, o objetivo do estudo é apresentar o desenvolvimento de uma ferramenta online que reúne uma enorme quantidade de informações para fácil acesso dos usuários que têm interesse em monitorar os diferentes padrões de teleconexão. Para isso, índices climáticos disponibilizados por diferentes centros de meteorologia são utilizados. Além disso, dois novos índices foram criados e implementados. A metodologia conta com 0 uso de linguagem de programação em web para sua implementação e para promover a visualização dos índices climáticos, mapas das variáveis atmosféricas, animações, análises estatísticas, bem como outros produtos. A ferramenta está disponível em: https://meteorologia.unifei.edu.br/teleconexoes/. Por fim, ressalta-se que a ferramenta foi projetada para ser automática e mensalmente atualizada.
Citation/Citação: Souza, C., \& Reboita, M. S. (2021). Ferramenta para o Monitoramento dos Padrões de Teleconexão na América do Sul. Terræ Didatica, 17(Publ. Contínua), 1-13, e021009. doi: 10.20396/td.v17i0.8663474.

Keywords: Visual and numerical tools. Indices. Statistical analysis. Seasonal Climate Forecast.

Palavras-chave: Ferramentas visuais e numéricas. Índices. Análise estatística. Previsão Climática Sazonal.

\section{Manuscript/Manuscrito:}

Received/Recebido: 02/12/2020

Revised/Corrigido: 25/01/2021

Accepted/Aceito: 22/02/2021

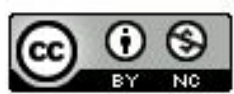

\section{Introdução}

As teleconexões estão associadas a perturbações na atmosfera que ocorrem num dado local e afetam o clima de regiões distantes no globo (Liu \& Alexander, 2007, Reboita et al., 2021). Muitos dos padrões de teleconexão se estabelecem associados às anomalias de temperatura da superfície do mar (TSM). Um dos casos mais amplamente conhecidos é o fenômeno El Niño-Oscilação Sul (ENOS), que na sua fase quente, isto é, quando há anomalias positivas (negativas) de TSM nos setores central e leste do oceano Pacífico Tropical, perturba a circulação atmosférica causando excesso (déficit) de precipitação no sudeste da América do Sul e déficit (excesso) no litoral norte das regiões norte e nordeste do Brasil e no interior da Amazônia (Andreoli et al., 2017, Reboita et al., 2021).
Como as anomalias ocorrem em intervalos de 2 a 7 anos, se diz que o ENOS é um fenômeno de escala interanual. Entretanto, os padrões de teleconexão possuem diferentes escalas temporais como, por exemplo, a intramensal (Modo Anular Sul), intrasazonal, (Oscilação de Madden-Julian), decadal (Oscilação Decadal do Pacífico) etc.

Quando se conhece os padrões anômalos de TSM, pode-se inferir sobre a presença de distintos modos de teleconexões e os prováveis impactos em diferentes locais do globo. Por isso, o conhecimento dos padrões de teleconexão ajuda a previsão climática sazonal. Esse tipo de previsão é um dos maiores desafios da atualidade. No Brasil, a previsão climática sazonal é realizada por grupos de pesquisadores que analisam as saídas de diferentes modelos numéricos de clima bem como utilizam 
o conhecimento dos padrões de teleconexão para fornecer prognósticos sazonais. Um grupo que realiza a tarefa é o Grupo de Estudos Climáticos da Universidade de São Paulo (www.grec.iag.usp. br). Entretanto, os padrões de teleconexão não são importantes apenas para a área das Ciências Atmosféricas, mas sim para a Física, Oceanografia, Biologia, Geografia, Geologia e Geociências, de forma geral.

Para o monitoramento dos padrões de teleconexão são utilizados índices baseados em diferentes variáveis atmosféricas e oceânicas. Esses índices são fornecidos (e atualizados) por diferentes centros de meteorologia nacionais e internacionais e, portanto, se encontram em bancos de dados distintos (espalhados pela internet), o que demanda tempo para organização das informações para discussões climáticas. Diante da necessidade de se ter informações rápidas e úteis para a previsão climática sazonal, este trabalho tem como objetivos: (a) descrever o desenvolvimento de uma ferramenta em que as informações são extraídas de diversos bancos de dados, processadas e apresentadas de forma visual e intuitiva aos usuários, que podem explorar os dados interativamente e obterem as relações entre as teleconexões e o clima de diferentes lugares; (b) mostrar ao usuário como navegar pela ferramenta e (c) apresentar a metodologia de dois novos índices criados para o monitoramento climático do sul e sudeste do Brasil. A ferramenta online (website) é chamada de "Ferramenta para o Monitoramento dos Padrões de teleconexão (FMPT)" e está disponível em https://meteorologia.unifei. edu.br/teleconexoes/.

\section{Índices de Teleconexões}

\section{Visão Geral}

As teleconexões são padrões de variabilidade natural do clima podendo ser identificadas a partir de técnicas estatísticas, como de função ortogonal empírica (EOF), agrupamento e a correlação temporal (Wilks, 2011, Wallace \& Gutzler, 1981). A técnica de correlação consiste na correlação de Pearson da série temporal de uma determinada variável com as séries temporais em pontos de grade de outra variável. Com isso, são obtidos padrões representados no espaço. O método de EOF envolve o cálculo de autovalores e autovetores e também permite encontrar padrões com maior variabilidade nos campos atmosféricos (Kutzbach, 1967). Já o método de análise de cluster tem sido menos utilizado para identificar padrões de teleconexão em escala global. A técnica comumente usada é a K-means clustering (Cazes-Boezio et al., 2003, Cozannet et al., 2011).

Uma vez conhecido os padrões de teleconexão, esses podem ser sintetizados e monitorados através de índices calculados com diferentes variáveis atmosféricas e oceânicas. Em geral, o sinal do índice revela o padrão da atmosfera e/ou oceano. Novamente, se utiliza do fenômeno ENOS para exemplificar a informação física de um índice. Para o caso do ENOS, anomalias de TSM são calculadas em setores específicos do oceano Pacífico Tropical. Quando no setor leste predominam águas mais quentes do que a média climatológica, o índice terá valor positivo, e se a anomalia quente persiste por uma sequência de meses, tem-se o estabelecimento do fenômeno El Niño. Aqui não será abordada a metodologia do cálculo dos índices de teleconexões, apenas será apresentada uma breve definição e explicação do que indica cada fase dos índices apresentados na FMPT. Para maiores detalhes metodológicos, recomenda-se o estudo de Reboita et al. (2021) e a própria FMPT (https://meteorologia.unifei.edu.br/teleconexoes/).

Ao longo dos anos, vários conjuntos de dados com diferentes frequências (diária, pêntada, mensal, trimestral e sazonal) têm sido aplicados para a obtenção dos índices de teleconexões. Para os índices utilizados neste trabalho (Fig. 1) será apresentada uma breve definição e a explicação do que cada fase dos índices indica.

\section{Modo Anular Sul (Southern Annular Mode)}

O Modo Anular Sul (SAM em inglês) é uma oscilação caracterizada por anomalias de sinais opostos entre os arredores da Antártica e as latitudes de $45^{\circ} \mathrm{S}$ (Thompson \& Wallace, 1998). A NOAA define o índice do SAM com base na técnica EOF. A fase positiva (negativa) é caracterizada por anomalias negativas (positivas) de altura geopotencial em $700 \mathrm{hPa}$ nos arredores da Antártica e positiva (negativa) nas latitudes médias. Isso afeta, por exemplo, a frequência de ciclones como mostrado por Reboita et al. (2015). Detalhes sobre a metodologia são providos em: https://www.cpc.ncep. noaa.gov/products/precip/CWlink/daily_ao_index/ history/method.shtml. 


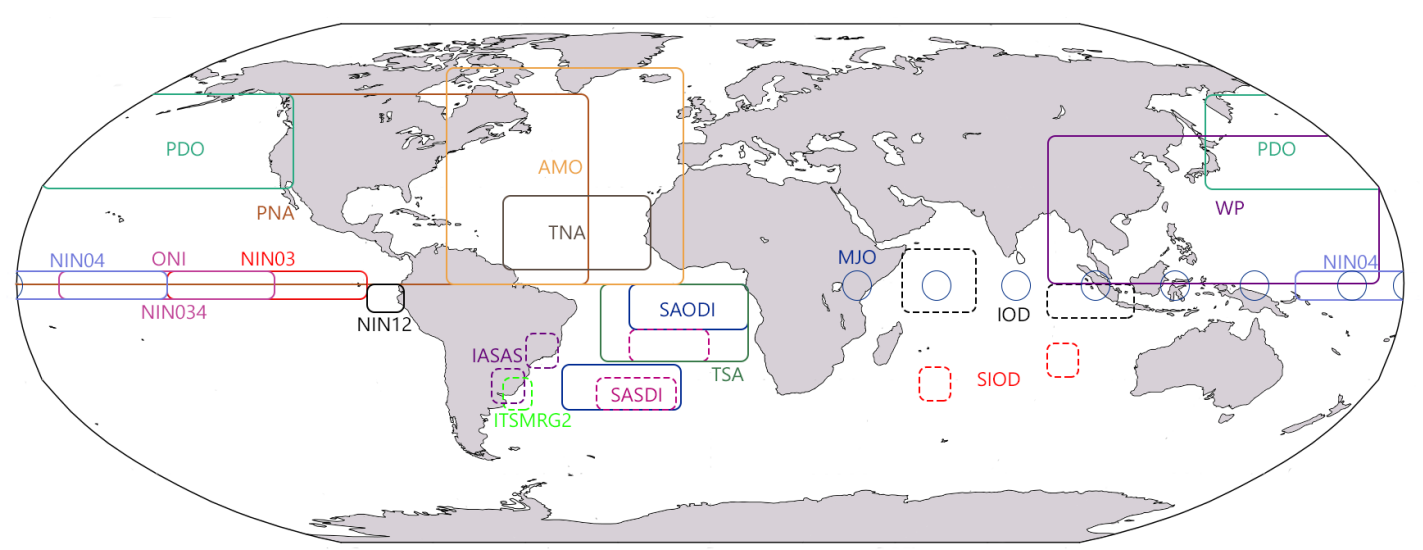

Figura 1. Área de desenvolvimento e/ou usada para identificação dos padrões de teleconexão que estão incluídos na FMPT

\section{Modo Anular do Ártico (Arctic Oscillation)}

O Modo Anular do Ártico (AO em inglês) é similar ao SAM, mas ocorre no Hemisfério Norte. A metodologia para calcular o índice também é similar à aplicada ao Hemisfério Sul e é dada em: https://www.cpc.ncep.noaa.gov/products/precip/ CWlink/daily_ao_index/ao.shtml. Quando o índice está em sua fase positiva, os ventos são mais intensos ao redor do polo e se tornam mais fracos na fase negativa.

\section{Padrão Pacífico-América do Norte (Pacific-North American Pattern)}

O Pacific North American Pattern (PNA) é um dos modos mais importantes de variabilidade de baixa frequência nos extratrópicos do Hemisfério Norte. A metodologia do índice do PNA é apresentada em https://www.cpc.ncep.noaa.gov/data/teledoc/ pna.shtml. A fase positiva do PNA está associada a temperaturas mais altas do que a média na costa oeste dos Estados Unidos e no oeste do Canadá. Durante a fase negativa, a costa oeste dos Estados Unidos pode ter temperaturas relativamente baixas, enquanto o leste dos Estados Unidos apresenta temperaturas acima da média. O PNA pode interagir com outros padrões de teleconexão causando impactos no clima da América do Sul.

\section{Padrão Pacífico-América do Sul (Pacific - South American Pattern)}

O Pacific South American Pattern (PSA) é similar ao PNA, mas ocorre no Hemisfério Sul. Quando a análise de EOF é aplicada às anomalias de altura geopotencial, o primeiro modo de variabilidade obtido é o SAM, enquanto que o segundo e o terceiro correspondem aos padrões PSA1 e PSA2 (Mo \& Higgins, 1998, Kidson, 1999, Mo, 2000). Tanto o PSA1 quanto o PSA2 estão associados à propagação de ondas de Rossby e com impactos na pressão atmosférica, principalmente ao sul de $20^{\circ} \mathrm{S}$, na América do Sul, favorecendo ou desfavorecendo a precipitação. Até o momento, os autores do presente estudo não conhecem nenhum centro que disponibiliza os índices do PSA1 e PSA2. Por esse motivo, tais índices também foram implementados na FMPT. A Figura 2 mostra a fase positiva dos padrões PSA1 e PSA2.
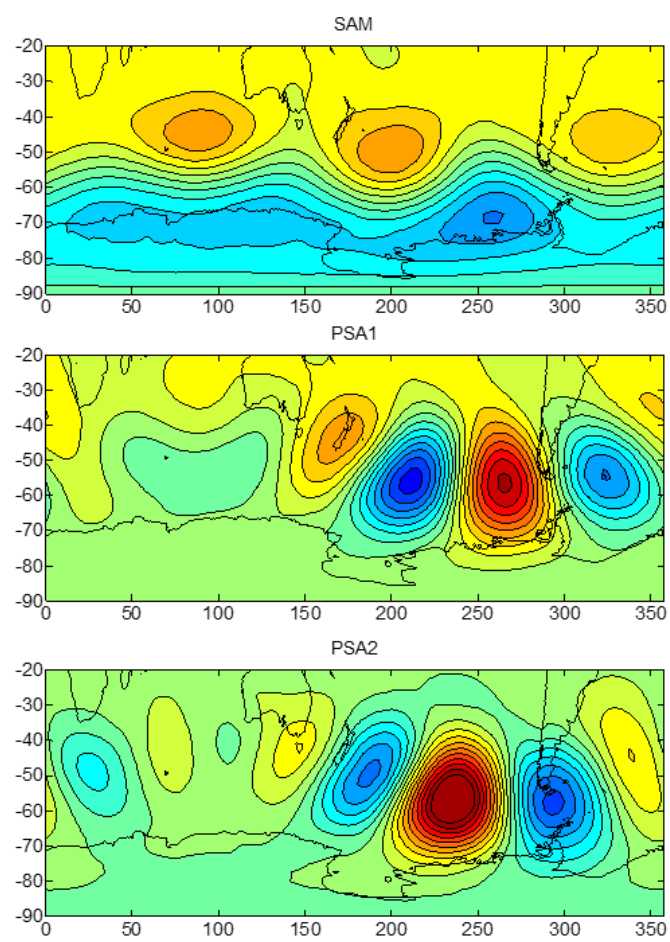

Figura 2. Fase positiva dos padrões SAM, PSA1 e PSA2 obtidos com a técnica de EOF e dados de anomalia de altura geopotencial em $700 \mathrm{hPa}$ 
A metodologia para obtenção dos índices PSA1 e PSA2 segue, em grande parte, a da NOAA (https://www.cpc.ncep.noaa.gov/products/precip/ CWlink/daily_ao_index/history/method.shtml) já que o primeiro modo de variabilidade obtido na técnica de EOF é o SAM. Primeiramente, tem-se que identificar os padrões PSA1 e PSA2 nos dados atmosféricos. Para isso, são utilizados dados das 1200 UTC (uma vez que tem maior número de observações da atmosfera assimiladas nas análises atmosféricas) de altura geopotencial em 700 hPa, no período de 2000 a 2020 , da região entre $20^{\circ} \mathrm{S}$ e $90^{\circ} \mathrm{S}$ para todas as longitudes do globo. A resolução horizontal da ERA5 é de $0,25^{\circ} \times 0,25^{\circ}$, mas para estudos de grande escala deve-se utilizar uma resolução mais grosseira para os padrões não serem afetados por fenômenos de mesoescala, por exemplo. Assim, os dados são interpolados com a técnica bi-linear para a grade de $2,5^{\circ} \times 2,5^{\circ}$. O passo seguinte é remover o ciclo anual dos dados. Isso é feito através da diferença entre o dado diário e a média climatológica do referente mês. O resultado desse processo também pode ser considerado como anomalia. De posse dos dados diários de anomalia (A), aplica-se a técnica de EOF. Aqui a técnica usou como base o método da singular value decomposition (SVD) para obtenção das matrizes de autovalores (D) e autovetores (U) conforme indicado por Green (1978). A SVD decompõe uma matriz em outras três, A = U D UU, sendo UU também uma matriz dos autovetores. Fazendo: $F=U^{1 / 2}$ obtêm-se as componentes principais que também são chamadas de leading modes ou component loadings. A variância explicada por cada componente principal (no caso, o SAM e o PSA1 e PSA2) é dada por $\mathrm{D}^{2} /(\mathrm{m}-1)$ onde $m$ é o número de linhas da matriz A. As componentes principais $(\mathrm{CP})$ podem ser pensadas como os padrões que mais se repetem num conjunto de dados. Considerado a altura geopotencial, a CP1 é a SAM, a CP2 é o PSA1 e a CP3 é o PSA2 (mostrados na Figura 2).

Imagine que se tem os dados diários de um determinado dia e se quer saber se há o padrão PSA1 e/ou o PSA2, como se obtém a informação? Para isso, pode-se calcular a anomalia da altura geopotencial no dado dia e a correlação espacial com a CP2 e com a CP3. Suponha que se calculou a correlação com a CP2 e se obteve um valor próximo de 1, isso indica que no dado dia predomina a fase positiva do PSA1 como mostrado na Figura 2. Se o valor for próximo de 1, mas negativo, a fase da PSA1 será negativa, ou em outras palavras, onde tem sinal positivo na Figura 2, ele torna-se negativo e vice-versa. Se a correlação for próxima de zero, isso indica que não há ocorrência do padrão PSA1.

A FMPT é utilizada para calcular a correlação entre as anomalias diárias da altura geopotencial e as CPs e para disponibilizar o índice obtido.

\section{Índice Oceânico do Niño (Oceanic Niño Index)}

O Oceanic Niño Index (ONI) é o principal indicador usado no monitoramento de El Niño e La Niña. O índice é calculado na mesma região do Niño 3.4 (região destacada na cor rosa na Fig. 1) e utiliza anomalias de TSM. A metodologia do ONI é apresentada em: https://origin.cpc.ncep. noaa.gov/products/analysis_monitoring/ensostuff/ONI_v5.php. As condições de El Niño (fase positiva) estão presentes quando o ONI é igual ou maior do que $0.5^{\circ} \mathrm{C}$ por 5 períodos trimestrais consecutivos. As condições de La Niña (fase negativa) existem quando o ONI é igual ou menor do que $-0,5^{\circ} \mathrm{C}$ por 5 períodos trimestrais consecutivos.

\section{Índices de Temperatura da Superfície do Mar no Pacífico}

No oceano Pacífico Tropical, há quatro regiões definidas na literatura para monitorar a ocorrência de eventos El Niño (EN) e La Niña (LN). As regiões são chamadas de EN1+2, EN3, EN3.4 e EN4 (Fig. 1) e os índices (anomalias de TSM) obtidos nessas regiões recebem o mesmo nome delas. A região EN1+2 é a menor e mais oriental das quatro regiões e corresponde ao setor costeiro da América do Sul. Já a região EN3.4 é a área mais sensível ao monitoramento do ENOS (Trenberth, 1997). Ela também é a mesma empregada para o cálculo do ONI.

\section{Índice Multivariado do ENOS (Multivariate ENSO Index)}

O Multivariate ENSO Index (MEI) foi originalmente definido por Wolter e Timlin (1993) com base na técnica de componente principal não rotacionada aplicada à combinação de seis variáveis de superfície no oceano Pacífico Equatorial. As informações sobre o MEI estão disponíveis em https://psl.noaa.gov/enso/mei/. Na sua fase positiva, o índice representa condições de El Niño e, na negativa, de La Niña.

\section{Índice de Oscilação Sul (Southern Oscillation Index)}

O Southern Oscillation Index (SOI) indica uma oscilação na pressão atmosférica entre os setores 
leste e oeste do oceano Pacífico Tropical (Walker, 1928). Portanto, o índice é conhecido como a componente atmosférica do ENOS. O SOI é calculado como a diferença da pressão atmosférica ao nível médio do mar entre uma estação meteorológica localizada no Tahiti e outra em Darwin (Austrália), SOI = PTahiti - PDarwin. (https://www.ncdc. noaa.gov/teleconnections/enso/indicators/soi/). Quando o SOI é positivo (negativo) indica que a pressão atmosférica ao nível médio do mar é maior (menor) em Tahiti do que em Darwin. Quando o índice apresenta por uma sequência de meses valores negativos (positivos) isso está relacionado com eventos de El Niño (La Niña). Nota-se aqui, que o SOI tem sinal inverso aos índices calculados com TSM para indicar os eventos de El Niño e La Niña.

\section{Índice do Pacífico Oeste (Western Pacific Index)}

O Western Pacific (WP) é um modo de variabilidade de baixa frequência no setor oeste do oceano Pacífico Norte. O padrão WP emerge como o segundo ou terceiro modo principal de variabilidade nas anomalias mensais da pressão ao nível médio do mar (Barnston \& Livezey, 1987). A metodologia desse índice encontra-se em detalhes em https://www.cpc.ncep.noaa.gov/data/teledoc/ wp.shtml. Durante o inverno e a primavera boreal, o padrão consiste em um dipolo norte-sul de anomalias, com um centro localizado sobre a Península de Kamchatka e um outro centro de sinal oposto cobrindo porções do sudeste da Ásia e do Pacífico subtropical ocidental norte. Portanto, fases positivas ou negativas intensas desse padrão refletem as variações zonais e meridionais pronunciadas na localização e na intensidade das correntes de jatos sobre a região.

\section{Oscilação Decadal do Pacífico (Pacific Decadal Oscillation)}

A Pacific Decadal Oscillation (PDO) refere-se a variações na temperatura da superfície do mar no oceano Pacífico Norte (Mantua et al., 1997). A PDO possui uma fase quente e outra fria, que possuem implicações significativas no clima global, uma vez que afetam a atividade de ciclones, alteram o trajeto das correntes de jatos e favorecem episódios de secas e inundações em torno da bacia do Pacífico bem como em regiões remotas (Mantua \& Hare, 2002). A PDO pode intensificar ou diminuir os impactos do ENOS de acordo com sua fase. Se o ENOS e o PDO estiverem na mesma fase, acredita-se que os impactos do El Niño/La Niña possam ser fortalecidos e, quando em fases opostas, enfraquecidos. A metodologia do cálculo da PDO é apresentada em https://www.ncdc.noaa. gov/teleconnections/pdo.

\section{Oscilação Quase-Bienal (Quasi-Biennial Oscillation)}

A Quasi-Biennial Oscillation (QBO) é um dos fenômenos marcantes na estratosfera equatorial, pois está associada à ocorrência de fortes ventos zonais, contornando o globo, e que permanecem de leste ou oeste num período médio de 28 a 29 meses (Baldwin et al., 2001). Detalhes do índice da QBO são fornecidos em https://acd-ext.gsfc.nasa. gov/Data_services/met/qbo/qbo.html. Em sua fase positiva, a QBO indica ocorrência de ventos de oeste e em sua fase negativa, de leste.

\section{Oscilação de Madden-Julian (Madden-Julian Oscillation)}

A Madden-Julian Oscillation (MJO) é uma oscilação intrasazonal que tem como fonte as anomalias positivas de TSM no oceano Índico. A MJO se desloca para leste e seu ciclo se completa entre 60-90 dias (Madden \& Julian, 1972). Ao longo de um ciclo, a MJO é caracterizada por 8 fases (regiões circulares nas cores azuis na Fig. 1) que são indicativas da localização dos máximos de atividade convectiva. Nas fases 8,1 e 2 , há condições propícias à convecção no nordeste do Brasil. Para a identificação das fases da MJO é utilizada a combinação de dois índices conforme a metodologia de Wheeler e Hendon (2004). Esses índices estão disponíveis em http://www.bom.gov.au/climate/mjo/.

\section{Dipolo do Oceano Índico (Indian Ocean Dipole)}

O Indian Ocean Dipole (IOD) é definido por anomalias de TSM de sinais opostos entre a região oeste $\left(50^{\circ} \mathrm{E}-70^{\circ} \mathrm{E}\right.$ e $\left.10^{\circ} \mathrm{S}-10^{\circ} \mathrm{N}\right)$ e leste do oceano Índico $\left(90^{\circ} \mathrm{E}-110^{\circ} \mathrm{E}\right.$ e $\left.10^{\circ} \mathrm{S}-0^{\circ} \mathrm{N}\right)$. O índice é disponibilizado por https://psl.noaa.gov/gcos_wgsp/ Timeseries/Data/dmi.had.long.data e sua fase positiva é definida quando há anomalias positivas (negativas) de TSM no setor oeste (leste) do Oceano Índico.

\section{Dipolo do Oceano Índico Subtropical (Subtropical Indian Ocean Dipole)}

O Subtropical Indian Ocean Dipole (SIOD) difere do IOD, uma vez que a TSM apresenta anomalias no sentido meridional e em região mais subtropical. 
Portanto, o SIOD é definido por anomalias de TSM de sinais opostos entre a região de Madagascar e o oeste da Austrália (Behera \& Yamagata, 2001). O dipolo foi descrito primeiramente por Behera et al. (2000) a partir de estudos observacionais e de simulações numéricas que relacionavam as anomalias da TSM positiva no setor oeste do Índico subtropical com as anomalias de precipitação na África do Sul e Central. O índice é obtido pela diferença das anomalias de TSM em duas regiões do oceano Índico (Região 1: $55^{\circ} \mathrm{E}-65^{\circ} \mathrm{E}$ e $37^{\circ} \mathrm{S}-27^{\circ} \mathrm{S}$ e Região 2: $90^{\circ} \mathrm{E}-100^{\circ} \mathrm{E}$ e $28^{\circ} \mathrm{S}-18^{\circ} \mathrm{S}$ ) e em sua fase positiva é definido quando há anomalias positivas de TSM próximo a Madagascar. O índice é implementado no presente estudo já que não é disponibilizado por nenhum centro.

\section{Oscilação Multidecadal do Atlântico (Atlantic Multidecadal Oscillation)}

A Atlantic Multidecadal Oscillation (AMO) é uma variação da temperatura da superfície do mar no oceano Atlântico Norte. A oscilação se caracteriza por períodos com cerca de 40 anos com anomalias positivas de TSM, seguidos por períodos de aproximadamente 20 anos com anomalias negativas de TSM no Atlântico Norte (Enfield et al., 2001). A metodologia para obtenção da AMO é dada em https://www.psl.noaa.gov/data/timeseries/AMO. Quando o índice está em fase positiva (valores negativos), a TSM é anomalamente quente (fria) na maior parte do oceano Atlântico Norte (região na cor laranja na Fig. 1).

\section{Oscilação do Atlântico Norte (North Atlantic Oscillation)}

Walker \& Bliss (1932) identificaram três padrões de teleconexão chamados de Oscilação do Atlântico Norte (NAO), Oscilação do Pacífico Norte (NPO) e Oscilação do Sul (SOI). A NAO é caracterizada por anomalias de pressão ao nível médio do mar com sinais opostos entre a Islândia-Groenlândia e o Atlântico Norte subtropical. Detalhes do índice da NAO são fornecidos em: https://www.cpc.ncep.noaa.gov/products/precip/CWlink/pna/nao.shtml. Os valores positivos indicam o aumento da diferença de pressão entre as duas regiões, resultando em uma corrente de jato mais intensa no oceano Atlântico. Quando a NAO está em sua fase negativa, a baixa subpolar e a alta subtropical estão mais fracas que a média e a corrente de jato do Atlântico têm uma orientação mais oeste-leste.

\section{Índice do Atlântico Tropical Sul (Tropical Southern Atlantic Index)}

O Tropical Southern Atlantic Index (TSA) é um indicador da TSM no oceano Atlântico Tropical Sul. A metodologia desse índice foi proposta por Enfield et al. (1999) e é utilizada pela NOAA para o cálculo e disponibilização do índice (https:// stateoftheocean.osmc.noaa.gov/sur/atl/tsa.php). Valores positivos do TSA indicam que a TSM é anomalamente quente no oceano Atlântico Tropical Sul (região destacada na cor verde na Fig. 1) enquanto que valores negativos indicam anomalia fria.

\section{Índice do Atlântico Tropical Norte (Tropical Northern Atlantic Index)}

O Tropical Northern Atlantic Index (TNA) é um indicador da temperatura da superfície do mar no oceano Atlântico Tropical Norte. Assim, como o TSA, o TNA foi proposto por Enfield et al. (1999) e é disponibilizado em https://stateoftheocean.osmc. noaa.gov/sur/atl/tna.php. Valores positivos do TNA indicam que a TSM é anomalamente quente no oceano Atlântico Tropical Norte (região destacada na cor marrom na Fig. 1) enquanto valores negativos indicam anomalia fria.

\section{Dipolo do Atlântico Sul (Southern Atlantic Dipole)}

O Southern Atlantic Dipole (SAD) corresponde a anomalias de TSM de sinais opostos no oceano Atlântico Sul entre as latitudes do sul do Brasil e Uruguai e o setor mais tropical desse oceano. $\mathrm{Na}$ literatura, há dois índices para o SAD: o South Atlantic Subtropical Dipole index (SASDI), que é o definido por Morioka et al. (2011), e o South Atlantic Ocean Dipole index (SAODI; http:// ljp.gcess.cn/dct/page/65592), que é definido por Nmanchi et al. (2011). Na Figura 1 são indicadas em cor rosa tracejada as áreas usadas no cálculo do SASDI e em azul escuro no cálculo do SAODI.

Como esses dois índices não estão sendo atualizados/disponibilizados pelos autores que os definiram, a metodologia para obtenção deles foi implementada e os resultados são apresentados na FMPT. Para o cálculo dos índices é utilizada a TSM do Extended Reconstructed Sea Surface Temperature (ERSST) versão 5 (Huang et al., 2017). Tanto para o SASDI quanto para o SAODI, é calculada a diferença entre a área mais tropical e a subtropical. 


\section{Índice do Anticiclone Subtropical do Atlântico Sul (IASAS)}

O Anticiclone Subtropical do Atlântico Sul (ASAS) influencia a precipitação ao longo do leste da América do Sul (Di Luca et al., 2006, Reboita et al., 2019, Garbarini et al., 2019): no verão seus ventos contribuem para o transporte de calor e umidade para o continente favorecendo a chuva; já no inverno, atua dificultando a passagem de frentes frias e a organização da convecção. Entretanto, há períodos em que o ASAS adquire características anômalas. Por exemplo, no verão de 2014, o ASAS adquiriu características típicas do inverno, que ajudaram na escassez hídrica no sudeste do Brasil (Coelho et al., 2016). Quando isso ocorre, a região sudeste do Brasil (com predomínio de anomalia positiva de pressão atmosférica) passa por um período seco, enquanto a região sul (com predomínio de anomalia negativa de pressão atmosférica), por um período chuvoso. Com a presença de anomalias de alta pressão em superfície na região sudeste, o jato de baixos níveis a leste dos Andes se dirige para a região sul, contribuindo para a ocorrência de precipitação no sul. A explanação torna evidente que o monitoramento das anomalias de pressão atmosférica nas regiões sul e sudeste do Brasil são relevantes. Para monitorar o ASAS, a Dra. Michelle Reboita, durante as reuniões climáticas do Grupo de Estudos Climáticos (GrEC) da USP, propôs um índice que se encontra implementado na FMPT. O índice do ASAS (IASAS) é obtido computando-se a diferença das anomalias mensais de pressão atmosférica ao nível médio do mar entre a região sudeste $\left(25^{\circ} \mathrm{S}-15^{\circ} \mathrm{S} ; 50^{\circ} \mathrm{W}-40^{\circ} \mathrm{W}\right)$ e sul $\left(37.5^{\circ} \mathrm{S}-27.5^{\circ} \mathrm{S} ; 60^{\circ} \mathrm{W}-50^{\circ} \mathrm{W}\right)$ do Brasil, como mostra a Figura 1 (cor roxa tracejada). Quando o índice é positivo, a pressão atmosférica ao nível do mar está maior na região sudeste do Brasil. O índice é computado usando dados de pressão ao nível médio do mar da reanálise ERA 5 (Hersbach et al., 2020). Além da disponibilização do IASAS mensal, a FMPT também fornece a opção de índice diário.

\section{Índice de TSM na RG2 (ITSMRG2)}

O GrEC/USP, desde a década de 2000, registra a presença de anomalias de TSM na região entre o Uruguai e o extremo sul do Brasil, que é conhecida como região ciclogenética RG2 nos estudos de Reboita et al. (2010, 2019) e de Jesus et al. (2020). Exemplos de episódios de anomalias quentes na referida região são mostrados na Figura 3. A fim de monitorar as anomalias na RG2, foi implementado na FMPT o cálculo das anomalias na região de $40^{\circ} \mathrm{S}-30^{\circ} \mathrm{S}$ e $57^{\circ}-47^{\circ} \mathrm{W}$ (destacada na cor verde clara tracejada na Fig. 1). A TSM utilizada é a Extended Reconstructed Sea Surface Temperature (ERSST) versão 5 (Huang et al., 2017). Quando o índice é negativo (positivo) indica águas mais frias (quentes) do que a climatologia.

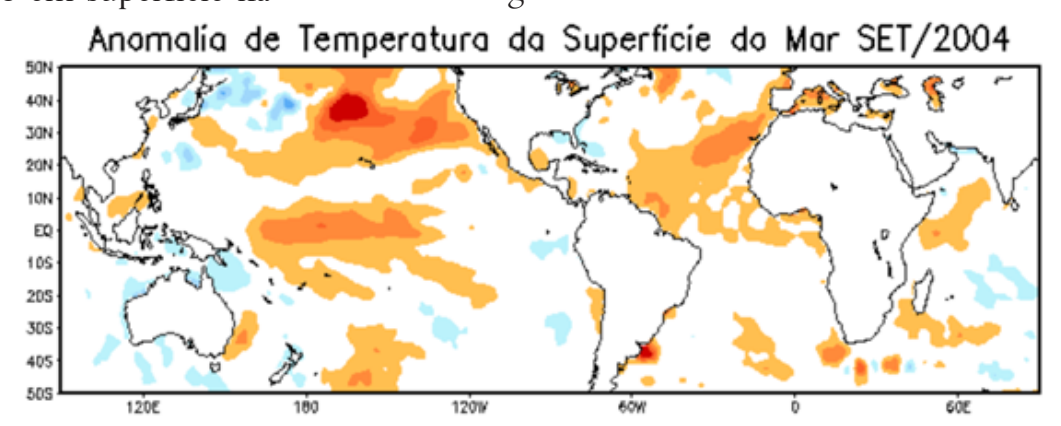

Anomalio de Temperaturo do Superficie do Mar SEP2009

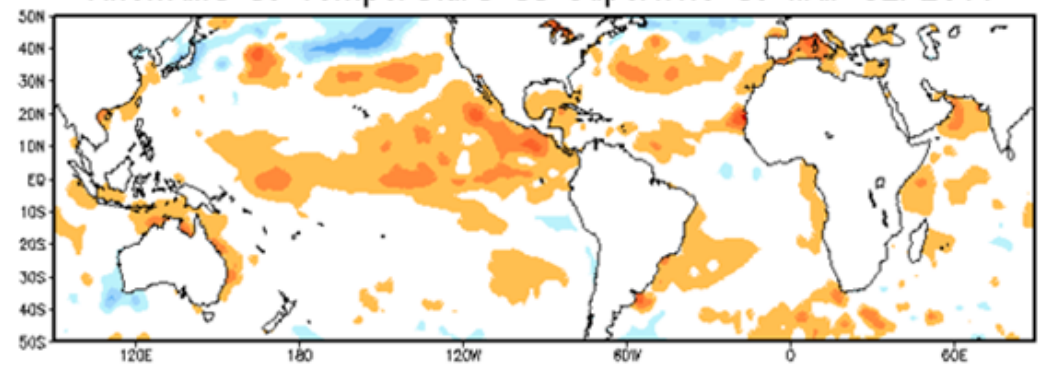

Anomalia de Temperatura do Superficie do Mar SEP2018

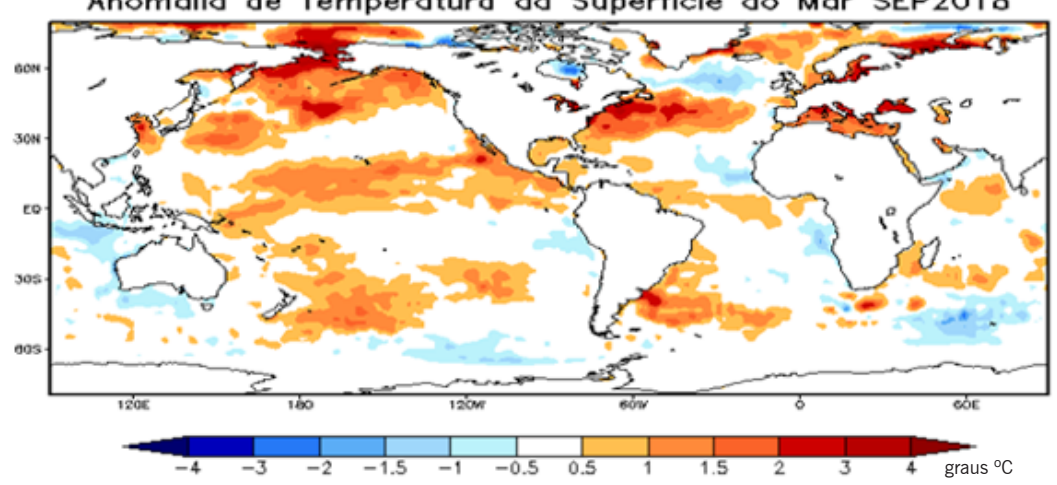

Figura 3. Exemplos de anomalias mensais de TSM. Fonte: CPTEC/INPE 


\section{Metodologia}

Para o desenvolvimento da FMPT há várias etapas que incluem desde a automatização da coleta de dados, processamento de figuras e índices, pós-processamentos, ferramentas web entre outras. Assim, são utilizadas diversas linguagens de programação, cada uma com seu propósito, como por exemplo: PHP para manipulação das séries temporais dos índices climáticos e texto, o Javascript para manipular os objetos orientados, geração de mapas e gráficos entre outros; o CSS para o design da ferramenta e o SQL para o gerenciamento de banco de dados e alguns frameworks.

A Figura 4 mostra o fluxograma de desenvolvimento da FMPT. Para o armazenamento dos índices climáticos (Tab. 1) foi utilizado o SQL (Structured Query Language) que é uma linguagem de gerenciamento do banco de dados. Ela permite a consulta de um grande volume de dados em milésimos de segundos, dessa maneira é possível obter vários cálculos estáticos utilizando todo o período histórico dos dados em tempo real pelos usuários no website. No banco de dados da página web, o período disponível de cada índice varia, como mostrado na Tabela 1.

A etapa seguinte consiste no processamento e cálculos dos índices das teleconexões que não estão disponíveis para obtenção nos centros de meteorologia (PSA1, PSA2, IASAS, ITSMRG2, SAODI e SASDI). A página também foi implementada de forma a permitir a visualização dos valores mensais dos índices (e para alguns índices, os valores diários) bem como os mapas das variáveis atmosféricas. A automação da execução desses processos é independente para cada índice, pois os diferentes centros internacionais atualizam e disponibilizam suas informações em períodos distintos. Por esse motivo, a FMPT desenvolvida faz a checagem a cada 10 dias para acrescentar os novos dados mensais. Após cada processamento, os resultados são enviados a um servidor online da Universidade Federal de Itajubá. Dessa forma, os programas de pós-processamento podem acessar e
Tabela 1. Índices climáticos utilizados e descrição de suas fontes

\begin{tabular}{|c|c|c|}
\hline Índices & Fontes & Banco de dados \\
\hline $\mathrm{AAO}$ & NOAA/CPC & 01/01/1979 - Presente \\
\hline $\mathrm{AMO}$ & NOAA/PSL & 01/01/1948 - Presente \\
\hline $\mathrm{AO}$ & NOAA/PSL & 01/01/1950 - Presente \\
\hline EN03 & NOAA/CPC & 01/01/1982 - Presente \\
\hline EN04 & NOAA/CPC & 01/01/1982 - Presente \\
\hline EN12 & NOAA/CPC & 01/01/1982 - Presente \\
\hline EN34 & NOAA/CPC & 01/01/1982 - Presente \\
\hline MEI & NOAA/PSL & 01/01/1979 - Presente \\
\hline $\mathrm{NAO}$ & NOAA/PSL & 01/01/1950 - Presente \\
\hline ONI & NOAA/PSL & 01/01/1950 - Presente \\
\hline $\mathrm{PDO}$ & NOAA/NCDC & 01/01/1854 - Presente \\
\hline PNA & NOAA/PSL & 01/01/1950 - Presente \\
\hline QBO & NOAA/PSL & 01/01/1948 - Presente \\
\hline SOI & NOAA/NCDC & 01/01/1951 - Presente \\
\hline TNA & NOAA/PSL & 01/01/1948 - Presente \\
\hline TSA & NOAA/PSL & 01/01/1948 - Presente \\
\hline WP & NOAA/PSL & 01/01/1950 - Presente \\
\hline MJO & BUREAU/AU & 01/06/1974 - Presente \\
\hline IOD & NOAA/WGSP & 01/01/1870 - Presente \\
\hline SASDI & Produzido/Gerado & 01/01/1979 - Presente \\
\hline SAODI & Produzido/Gerado & 01/01/1979 - Presente \\
\hline IASAS & Produzido/Gerado & 01/01/1979 - Presente \\
\hline ITSMRG2 & Produzido/Gerado & 01/01/1979 - Presente \\
\hline SIOD & Produzido/Gerado & 01/01/1979 - Presente \\
\hline PSA1 & Produzido/Gerado & 01/01/1979 - Presente \\
\hline PSA2 & Produzido/Gerado & 01/01/1979 - Presente \\
\hline
\end{tabular}

Tabela 2. Variáveis utilizadas para a geração dos mapas

\begin{tabular}{|c|c|c|}
\hline \multicolumn{3}{|c|}{ ERA 5} \\
\hline Variável & Unidade & Método \\
\hline \multirow{2}{*}{ Pressão Atmosférica } & \multirow{2}{*}{$\mathrm{hPa}$} & Média \\
\hline & & Anomalia \\
\hline \multirow{2}{*}{ Temperatura a 2 metros } & \multirow{2}{*}{${ }^{\circ} \mathrm{C}$} & Média \\
\hline & & Anomalia \\
\hline \multirow{2}{*}{ Temperatura em $850 \mathrm{hPa}$} & \multirow{2}{*}{${ }^{\circ} \mathrm{C}$} & Média \\
\hline & & Anomalia \\
\hline \multirow{2}{*}{ Temperatura em $500 \mathrm{hPa}$} & \multirow{2}{*}{${ }^{\circ} \mathrm{C}$} & Média \\
\hline & & Anomalia \\
\hline \multirow{2}{*}{ Altura Geopotencial em $850 \mathrm{hPa}$} & \multirow{2}{*}{$\mathrm{m}$} & Média \\
\hline & & Anomalia \\
\hline \multirow{2}{*}{ Altura Geopotencial em $500 \mathrm{hPa}$} & \multirow{2}{*}{$\mathrm{m}$} & Média \\
\hline & & Anomalia \\
\hline $\begin{array}{l}\text { Altura Geopotencial em 500-850 } \\
\qquad \mathrm{hPa}\end{array}$ & $\mathrm{m}$ & Diferença \\
\hline \multirow{2}{*}{ Temperatura da superfície do mar } & \multirow{2}{*}{${ }^{\circ} \mathrm{C}$} & Média \\
\hline & & Anomalia \\
\hline \multicolumn{3}{|l|}{$\mathrm{CPC}$} \\
\hline Variável & Unidade & Método \\
\hline \multirow{2}{*}{ Precipitação } & \multirow{2}{*}{$\mathrm{mm}$} & Média \\
\hline & & Anomalia \\
\hline
\end{tabular}




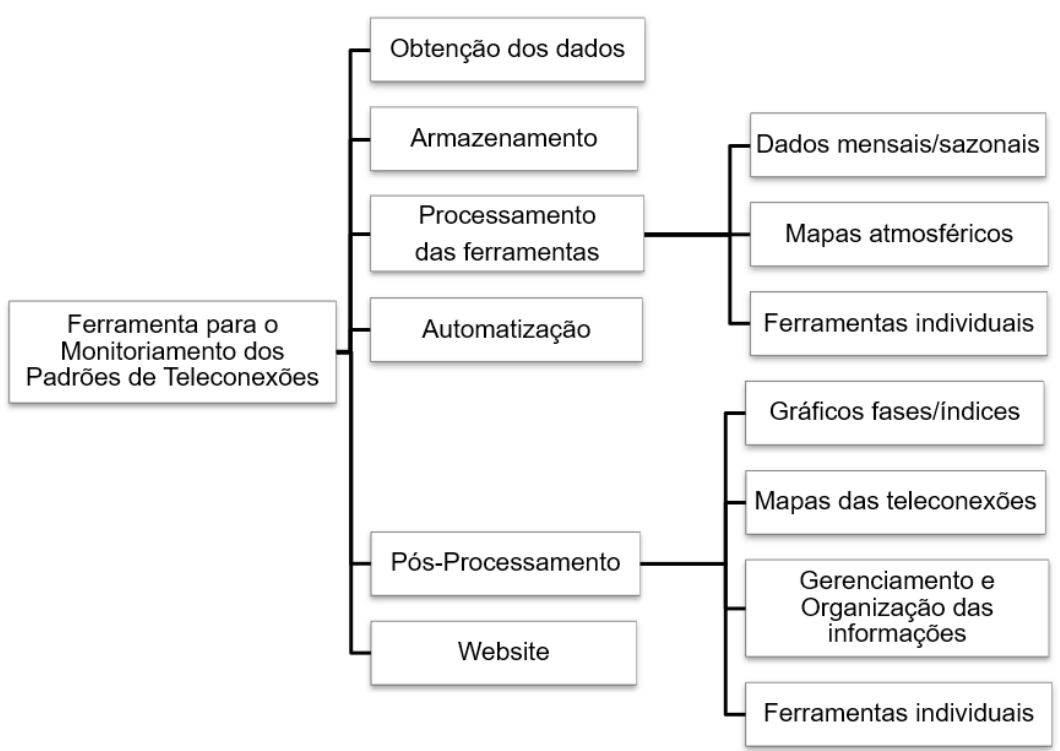

Figura 4. Esquema dos processos da FMPT

organizar as informações e ferramentas para os usuários.

O usuário pode gerar as figuras das variáveis descritas na Tabela 2. O processo está atualmente configurado para utilizar a reanálise ERA 5 (Hersbach et al., 2020) com resolução horizontal de $0,25^{\circ}$ e as análises de precipitação do CPC (Chen et al., 2008), com 0,5 de resolução horizontal. Os mapas das variáveis atmosféricas são gerados com a frequência temporal mensal e são disponibilizadas médias e anomalias calculadas de 1979 até o presente.

\section{Resultados e Discussão}

O objetivo principal deste trabalho é oferecer um produto funcional para auxiliar os usuários na previsão climática sazonal. Dessa forma, vários índices de teleconexões foram compilados numa página web desenvolvida, que também é chamada de FMPT, e está disponível no website do curso de Ciências Atmosféricas da UNIFEI: https://meteorologia.unifei.edu. $b r / t e l e c o n e x o e s$. Na sequência está apresentada uma descrição detalhada do website, o que ajuda a navegação por parte do usuário.

A página inicial da FMPT (Fig. 5) inicia apresentando: (a) uma síntese dos valores mensais atuais dos índices de tele- conexões; (b) três destaques para acesso de informações mais detalhadas: Análises das Teleconexões, Região das Teleconexões e Fases das Teleconexões; (c) opção teleconexão que permite a visualização das séries temporais e (d) uma opção para visualização das regiões utilizadas no cálculo dos índices de teleconexões.

Com relação ao item b, tem-se:

(1) Análise das Teleconexões: Quando o usuário acessa a opção Análise das Teleconexões na página inicial, irá encontrar uma lista
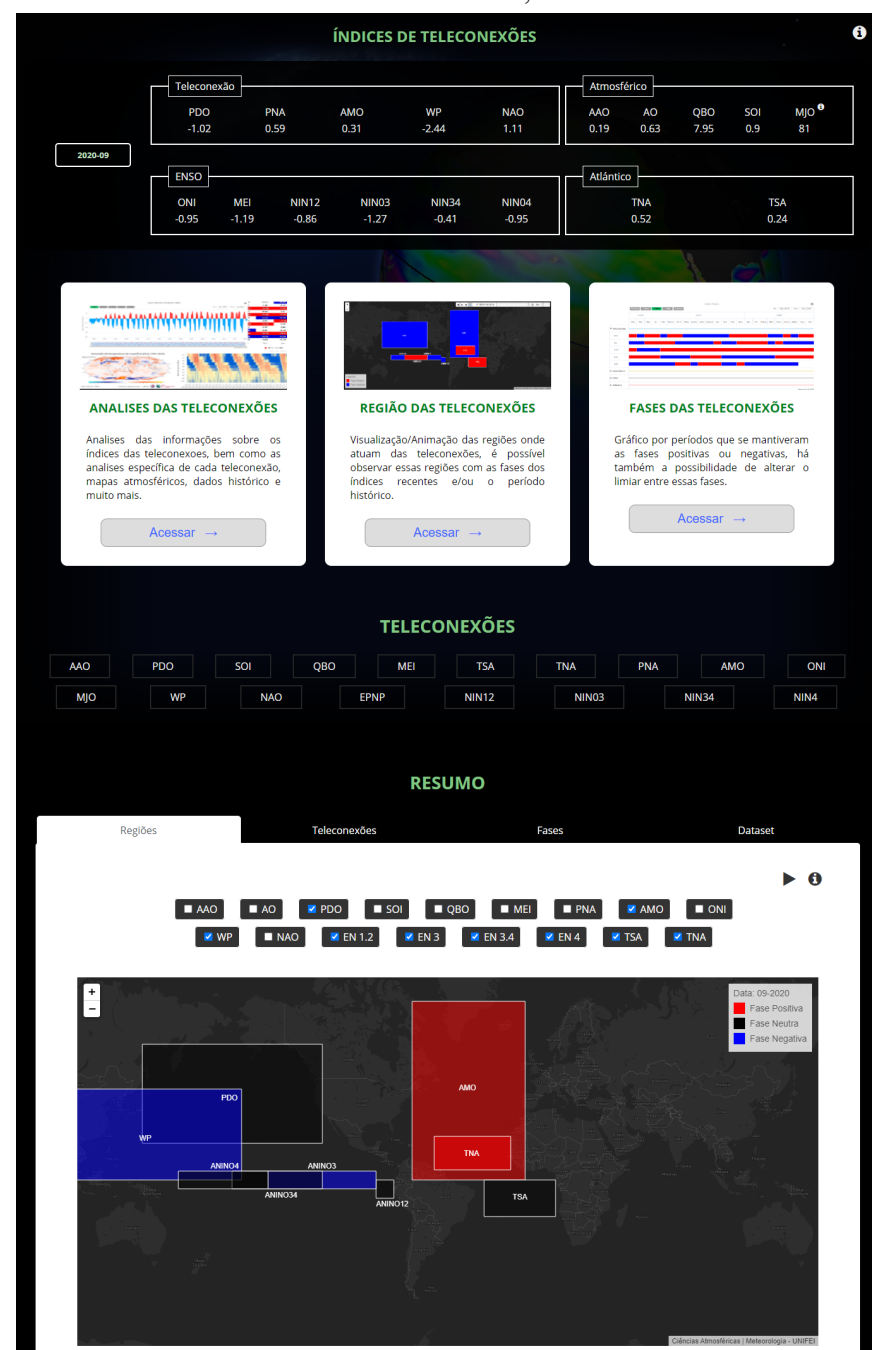

Figura 5. Página inicial do website

\begin{tabular}{c|c|c|c|c|c}
\hline (C) Terrae Didat. & Campinas, SP & v.17 & $1-13$ & e 021009 & 2021 \\
\hline
\end{tabular}




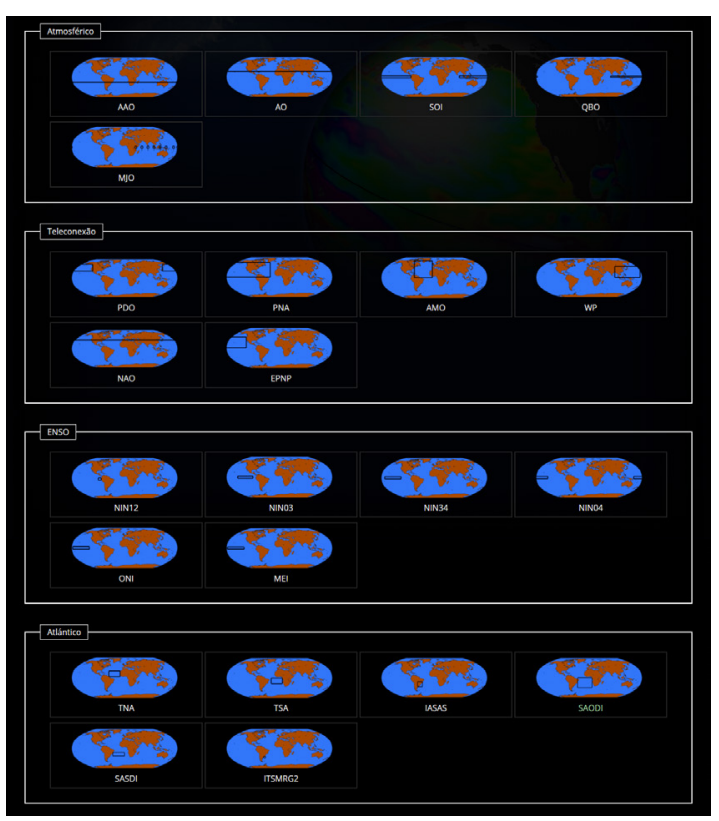

Figura 6. Página da seleção dos índices

com os índices de teleconexões (Fig. 6) agrupados nas categorias (i) atmosférico, (ii) teleconexões, (iii) ENSO, (iv) Atlântico e (v) Índico.

Ao escolher um índice, o usuário é levado a uma nova página (Fig. 7) que se inicia com um gráfico interativo e dinâmico com todo o período histórico do índice desejado e que poderá ser visualizado também por períodos específicos, como os últimos 30,10, 5 anos e/ou alternar entre qualquer período entre o começo e o final da série. O usuário também conseguirá exportar as informações nos formatos: png, jpg, csv, xls, pdf entre outros.

Nessa página ainda há 4 abas para navegação: visão geral, métodos, mapas e dados históricos (alguns índices mostram uma quinta ou sexta opção com informações adicionais). A aba visão geral fornece uma descrição do padrão de teleconexão escolhido e uma breve revisão da literatura. Nessa mesma aba ainda há uma ficha de detalhamento do índice de teleconexão, como, por exemplo, a fase atual, sua intensidade, gráficos mensais e anuais de sua variabilidade, cálculo estatísticos de correlação. A aba método apresenta a metodologia do cálculo de cada índice de teleconexão (Fig. 8).

A aba mapas (Fig. 9) é uma das partes mais interessantes da FMPT, pois permite que o usuário visualize mapas mensais da média ou da anomalia de diferentes variáveis atmosféricas (Tabela 2). Além disso, é possível escolher o mês desejado. Para a precipitação é utilizado o banco de dados do CPC (Chen et al., 2008) enquanto que para as demais variáveis, a reanálise ERA5 (Hersbach et

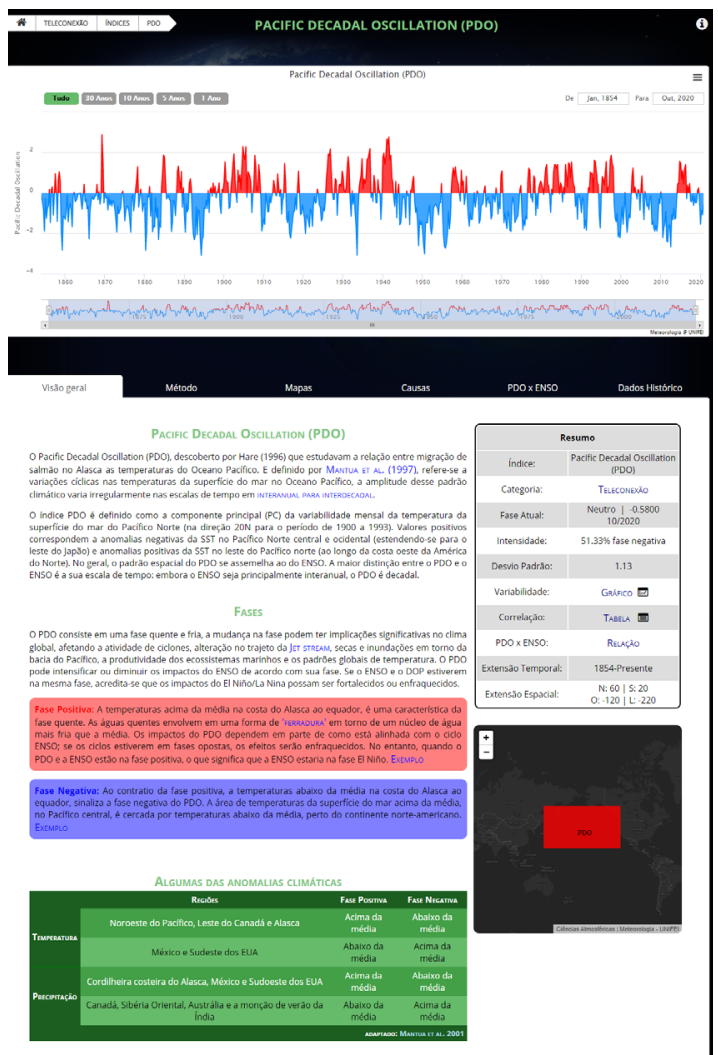

Figura 7. Página principal dos índices, mostrando o índice PDO

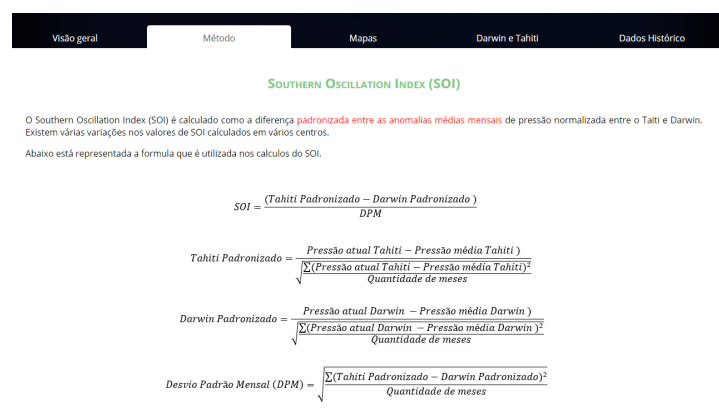

Figura 8. Aba dos métodos, na página principal dos índices, mostrando para o índice SOI
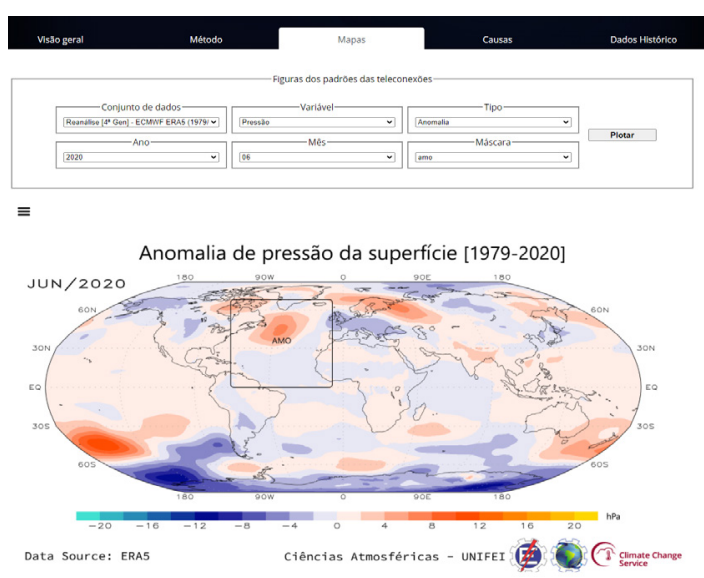

Figura 9. Mapa das condições atmosféricas

\begin{tabular}{c|c|c|c|c|c|}
\hline C Terrae Didat. & Campinas, SP & v.17 & $1-13$ & e021009 & 2021 \\
\hline
\end{tabular}




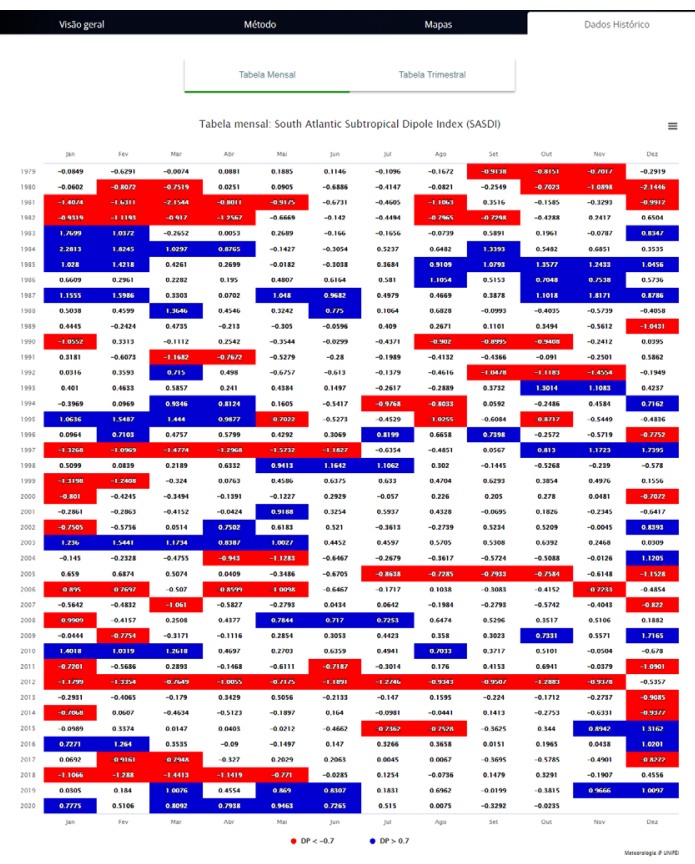

Figura 10. Visualização dos dados históricos aliza as fases dos índices de teleconexões (Fig. 12). Também há a possibilidade de o usuário alterar o limiar entre as fases, assim, podendo analisar períodos em que os índices de teleconexões ultrapassem a um determinado valor específico, por padrão o limiar é selecionado como zero e há opções de escolher entre o desvio-padrão de todo o período ou definir algum valor da escolha do usuário.

\section{Conclusões}

O estudo aplicou conhecimentos de diversas áreas, como Ciências Atmosféricas, Geografia, Estatística e Computação, para desenvolver uma ferramenta online para organização e visualização de informações sobre índices de teleconexões e padrão das variáveis atmosféricas a fim de facilitar as práticas de previsão climática sazonal. A ferramenta online foi denominada de Ferramenta para o Monitoramento dos Padrões de teleconexão (FMPT). Ao todo 26 índices de teleconexões foram al., 2020). A anomalia corresponde à média do mês escolhido menos a média climatológica e o período utilizado para esses cálculos é de 1979-2020.

Por fim, a aba dados históricos (Fig. 10) mostra uma tabela mensal e outra trimestral com os dados históricos de cada índice. Além disso, são destacados em cor azul os valores mais negativos que o desvio-padrão e em vermelho os maiores que o desvio-padrão. $\mathrm{O}$ usuário pode fazer o download da série histórica em vários formatos, como em csv, xls, pdf, png etc.

Para alguns índices, a FMPT possui aba adicional, como por exemplo, para os índices relacionados ao ENOS, em que suas séries temporais são mostradas concomitantemente.

(2) Região das Teleconexões: selecionando a opção o usuário é levado a uma nova janela onde é mostrado, em mapa, as regiões usadas no cálculo dos índices e é mostrada uma evolução temporal da fase do índice. (Fig. 11).

(3) Fases das Teleconexões: ao clicar nessa opção, o usuário visu-

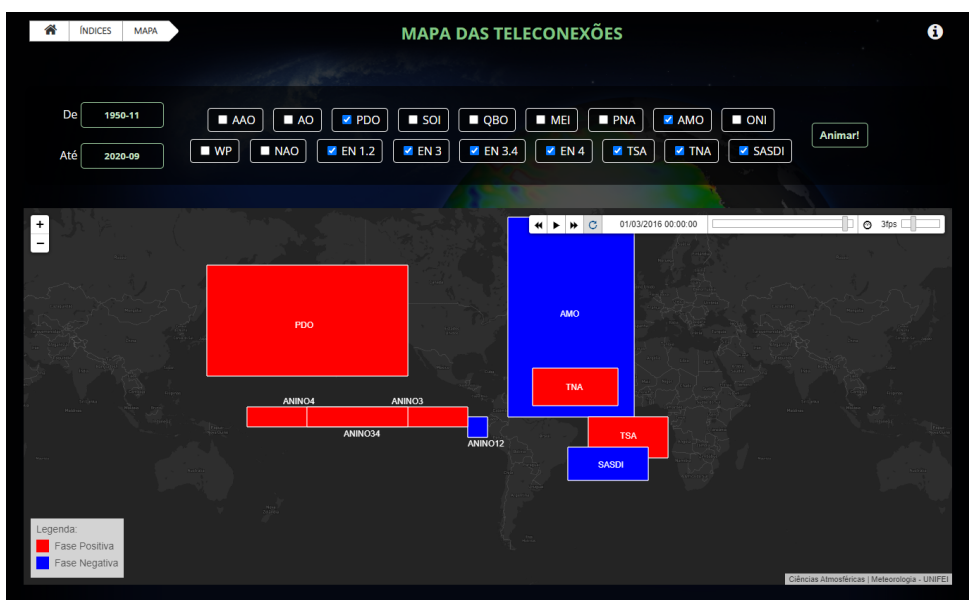

Figura 11. Ferramenta do mapa com animação das teleconexões

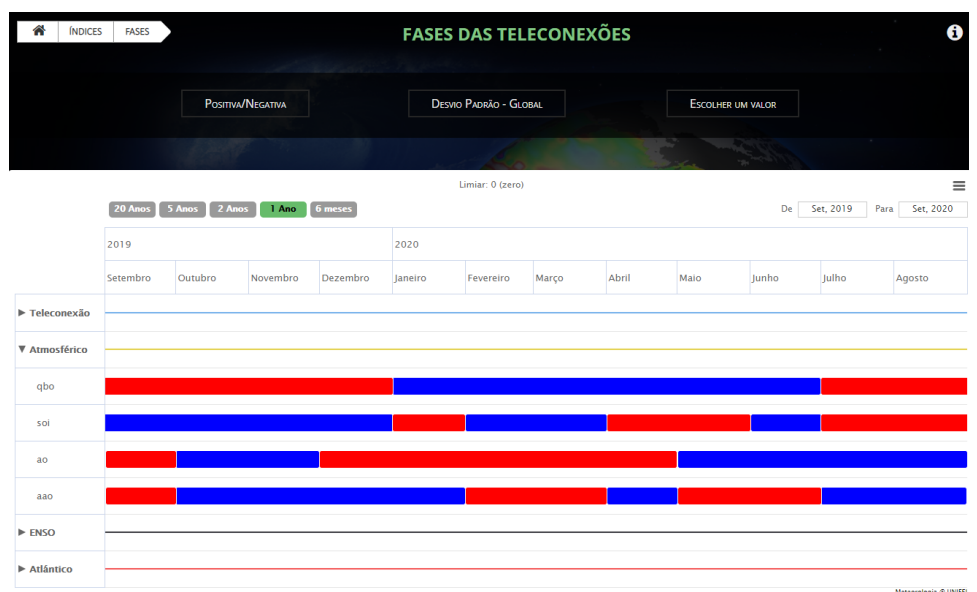

Figura 12. Página das fases dos índices 
implementados na FMPT, sendo 2 definidos exclusivamente para este trabalho (IASAS e ITSMRG2). Para isso, foram utilizadas várias técnicas de programação desde o processo de automatização para obter os dados, processamento de alguns índices, geração das ferramentas visuais como os gráficos interativos, mapas das teleconexões e figuras das variáveis atmosféricas até a organização final dos resultados para o usuário. Também foram empregados vários métodos estatísticos e comparativos para que outros usuários utilizem a ferramenta para realizar análises sobre as características do clima com os índices do passado e do presente.

A FMPT foi projetada para que receba, de forma simples, novos conjuntos de dados de índices de teleconexões e novas funcionalidades, permitindo a fácil implementação de novos índices e funções. Assim, será uma ferramenta em constante atualização para atender as necessidades de diversas áreas como Oceanografia, Geografia, Geociências etc.

\section{Agradecimentos}

Os autores agradecem aos centros de meteorologia que disponibilizam os dados utilizados no estudo e ao CNPq e FAPEMIG pelo apoio financeiro.

\section{Referências}

Andreoli, R.V., Oliveira, S.S., Kayano, M.T., Viegas, J., Souza, R. A. F., \& Candido, L. A. (2017). The influence of different El Niño types on the South American rainfall. Int. J. Climatology, 3, 1374-1390. doi: 10.1002/joc.4783.

Baldwin, M. P., Gray L. J., Dunkerton, T. J., Hamilton, K., Haynes, P. H., Randel, W. J, ... \& Takahashi, M. (2001). The quasi-biennial oscillation. Reviews of Geophysics, 39(2), 179-229. doi: 10.1029/1999RG000073.

Barnston, A. G., \& livezey, R. E. (1987). Classification, Seasonality and Persistence of Low Frequency Atmospheric Circulation Patterns. Monthly Weather Review, 115(6), 1083-1126. doi: 10.1175/1520-0493(1987)115<1083:CSAPOL > 2 $0 . \mathrm{CO} ; 2$

Behera, S. k., \& Yamagata, T. (2001). Subtropical SST dipole events in the southern Indian Ocean. Geophysical Research Letters, 28(2), 327-330. doi: 10.1029/2000GL011451.

Behera, S. k., Salvekar, P. S., \& Yamagata, T. (2000). Simulation of Interannual SST Variability in the Tropical Indian Ocean. Journal of Climate, 13(19), 34873499. doi: 10.1175/1520-0442(2000)013<3487:SO ISVI $>2.0 . \mathrm{CO} ; 2$

Cazes-Boezio, G., Robertson, A. W., \& Mechoso, C. R., (2003). Seasonal Dependence of ENSO Telecon- nections over SouthAmericaand Relationshipswith Precipitation in Uruguay. J. Climate, 16(8), 11591176. doi: 10.1175/1520-0442(2003)16<1159:SD OETO > 2.0.CO;2.

Chen, M., Shi, W., Xie, P., Silva, V. B. S., Kousky, V. E., Higgins, R. W., \& Janowiak, J. E. (2008). Assessing objective techniques for gauge-based analyses of global daily precipitation, J. Geophys. Res., 113, 1-13. doi: 10.1029/2007JD009132.

Coelho, C.A.S., Oliveira, C.P., Ambrizzi, T., Reboita, M. S., Carpenedo, B., Campos, J. L. P. S., ... \& Rehbein, A. (2016). The 2014 southeast Brazil austral summer drought: regional scale mechanisms and teleconnections. Clim. Dyn., 46, 3737-3752. doi: 10.1007/s00382-015-2800-1.

Cozannet, G. L., Lecacheux, S., Delvallee, E., Desramaut, N., Oliveros, C., \& Pedreros, R. (2011). Teleconnection Pattern Influence on Sea-Wave Climate in the Bay of Biscay. J. Climate, 24(3), 641652.doi: 10.1175/2010JCLI3589.1.

CPTEC/INPE. (2020). El Niño e La Niña. URL: http:// enos.cptec.inpe.br/. Acesso 20.11.2020.

Di Luca, A., Camilloni, I., \& Barros, V. (2006). Sea-Level Pressure Patterns In South America And The Adjacent Oceans In The IPCC Ar4 Models. $8^{\text {th }}$ International Conference on Southern Hemisphere Meteorology and Oceanography, 235-243.

Enfield, D. B., Mestas-Nuñez, A. M., \& Trimble, P. J. (2001). The Atlantic Multidecadal Oscillation and its relation to rainfall and river flows in the continental U.S. Geophysical research letters, 28(10), 78417848. doi: 10.1029/2000GL012745.

Enfield, D. B., Mestas-Nuñez, A. M., Mayer, D. A., \& Cid-Serrano, L. (1999). How ubiquitous is the dipole relationship in tropical Atlantic sea surface temperatures? J. Geophys Res., 104(4), 7841-7848. doi: 10.1029/1998JC900109.

Garbarini, E. M., González, M. H., \& Rolla, A. L. (2019). The influence of Atlantic High on seasonal rainfall in Argentina. Int. J. Climatology, 39(12), 4688-4702. doi: 10.1002/joc.6098.

Green, P. E. (1978). Analyzing Multivariate Data, Illinois: Dryden Press. 519p.

Hersbach, H., Bell, B., Berrisford, P., Berrisford, P., Hirahara, S., Horányi, A., ... \& Thépaut, J. (2020). The ERA5 global reanalysis. Q. J. R. Meteorol. Soc., 146, 1999-2049. doi: 10.1002/qj.3803.

Huang, B., Thorne, P. W., Banzon, V. F., Boyer, T., Chepurin, G. A., Lawrimore, J. H, ... Zhang, H. (2017). Extended Reconstructed Sea Surface Temperature, Version 5 (ERSSTv5): Upgrades, Validations, and Intercomparisons. Journal of Climate, 30(20), 8179-8205. doi: 10.1175/JCLI-D-16-0836.1.

Jesus, E. M. (2020). Ciclones e ciclones subtropicais sobre o sudoeste do Oceano Atlântico Sul: projeções climáticas e ventos associados. São Paulo: Universidade de São Paulo. 141p. (Tese Doutorado). doi: 10.11606/T.14.2020.tde-04092020-231228.

Kidson, J. W. (1999). Principal Modes of Southern Hemisphere low-frequency variability obtained from NCEP-NCAR reanalyses. J. Climate, 12(9), 2808-2830. doi: 10.1175/1520-0442(1999)012<2808:PMOSHL> 
2.0.CO;2.

Kutzbach, J. E. (1967). Empirical Eigenvectors of Sea-Level Pressure, Surface Temperature and Precipitation Complexes over North America. Journal of Applied Meteorology, 6(5), 791-802. doi: 10.1175/1520-0450(1967)006<0791:EEOSLP > 2 $.0 . \mathrm{CO} ; 2$.

Liu, Z. \& Alexander. M. (2007). Atmospheric bridge, oceanic tunnel, and global climatic teleconnections. Rev. Geophys, 45(2), 27-40. doi: 10.1029/2005RG000172.

Madden, R. A., \& Julian, P. R. (1972). Description of Global-Scale Circulation Cells in the Tropics with a 40-50 Day Period. J. Atmos. Sci., 29(6), 1109-1123. doi: 10.1175/1520-0469(1972)029<1109:DOGS $\mathrm{CC}>2.0 . \mathrm{CO} ; 2$.

Mantua, N. J. \& Hare, S. R. (2002). The Pacific Decadal Oscillation. Journal of Oceanography, 58, 35-44. doi: 10.1023/A:1015820616384.

Mantua, N. J., Hare, S., Zhang, Y., Wallace, J. M., \& Francis, R. C. (1997). A Pacific Interdecadal Climate Oscillation with Impacts on Salmon Production. Bulletin of the American Meteorological Society, 78(6), 1069-1080. doi: 10.1175/1520-0477(1997)078<1069:APICOW > 2 $.0 . \mathrm{CO} ; 2$.

Mo, K. C. \& Higgins. R. W. (1998). The PacificSouth American Modes and Tropical Convection during the Southern Hemisphere Winter. Monthly Weather Review, 126(6), 1581-1596. doi: 10.1175/1520-0493(1998)126<1581:TPSAMA > 2 .0.CO;2.

Mo, K. C. (2000). Relationships between LowFrequency Variability in the Southern Hemisphere and Sea Surface Temperature Anomalies. J. of Climate, 13(20), 3599-3610. doi: 10.1175/1520-0442(2000)013<3599:RBLFVI > 2 . $0 . \mathrm{CO} ; 2$.

Morioka, Y., Tozuka, T., \& Yamagata, T. (2011). On the Growth and Decay of the Subtropical Dipole Mode in the South Atlantic. J. Climate, 24(21), 5538-5554. doi: 10.1175/2011JCLI4010.1.

Nnamchi, H. C., Li, J., \& Anyadike, R. N. C. (2011). Does a dipole mode really exist in the South Atlantic Ocean?.J. Geophys. Res., 116(15), 442-457. doi: 10.1029/2010JD015579.

Reboita M. S., Ambrizzi T., Silva B. A., Pinheiro R. F., \& Rocha R. P. (2019). The South Atlantic Subtropical Anticyclone: Present and Future Climate. Frontiers in Earth Science, 7(8). 1-15. doi: 10.3389/feart.2019.00008

Reboita, M. S., Ambrizzi, T., Crespo, N. M., Dutra, L. M. M., Ferreira, G. W. S., Rehbein, A., Drumond, A., Rocha, R. P., \& Souza, C. A. (2021). Impacts of Teleconnection Patterns on South America Climate: a Review, Ann. NY Acad. Sci. (Aceito para publicação).

Reboita, M. S., Rocha, R. P., Ambrizzi, T., \& Gouveia, C. D. (2015). Trend and teleconnection patterns in the climatology of extratropical cyclones over the Southern Hemisphere. Climate Dynamics, 45, 1929-1944. doi: 10.1007/s00382-014-2447-3.

Reboita, M. S., Rocha, R. P., Ambrizzi, T., \& Sugahara, S. (2010). South Atlantic Ocean cyclogenesis climatology simulated by regional climate model (RegCM3). Climate Dynamics, 35, 1331-1347. doi: 10.1007/s00382-009-0668-7.

Thompson, D. W. J., \& Wallace, J. M. (1998). The Arctic oscillation signature in the wintertime geopotential height and temperature fields. Geophysical research letters, 25(9), 1297-1300. doi: 10.1029/98GL00950.

Trenberth, K. E. (1997). The Definition of El Niño. Bulletin of the American Meteorological Society, 78(12), 2771-2778. doi: 10.1175/1520-0477(1997)078<2771:TDOENO $>2.0 . \mathrm{CO} ; 2$.

Walker, G. T. (1928). World Weather. Quarterly Journal of the Royal Meteorological Society, 54(226), 79-87. doi: 10.1002/qj.49705422601.

Walker, G.T., \& Bliss, E.W. (1932). World Weather V. Memoirs of the Royal Meteorological Society, 4, 53-84.

Wallace, J. M., \& Gutzler, D. S. (1981). Teleconnections in the Geopotential Height Field during the Northern Hemisphere Winter. Monthly Weather Review, 109(4), 784-812. doi: 10.1175/1520-0493(1981)109<0784:TITGHF > 2 $.0 . \mathrm{CO} ; 2$.

Wheller, M. C., \& Hendon, H. (2004). An All-Season Real-Time Multivariate MJO Index: Development of an Index for Monitoring and Prediction. Monthly Weather Review, 132(8), 1917-1932. doi: 10.1175/1520-0493(2004)132<1917:AARMMI> 2.0.CO;2.

Wilks, D. S. (2011). Statistical Methods in the Atmospheric Sciences. Burlington, MA: Academic Press. 704p.

Wolter, K., \& Timlin. M.S. (1993). Monitoring ENSO in COADS with a Seasonally Adjusted Principal Component Index. 17th Climate Diagnostics Workshop, 52-57. 